\title{
Desempenho econômico da cultura do girassol em sistemas de agricultura familiar do sudeste paranaense
}

\author{
Economic performance of sunflower in family farming systems of southeastern Paraná, southern \\ Brazil
}

\author{
Maria de Fátima dos Santos Ribeiro ${ }^{\mathrm{I}}$ Augusta Pelinski Raiher ${ }^{\mathrm{II}}$
}

\section{RESUMO}

O objetivo deste trabalho foi avaliar o desempenho econômico da cultura do girassol em sistemas de produção familiar do sudeste paranaense. Foram avaliadas 26 situações culturais, as quais variaram quanto às condições edafoclimáticas, à intensidade de uso de mecanização, ao custo com fertilizantes e à cultivar utilizada. A Análise de Componentes Principais indicou que o desempenho econômico da cultura do girassol não esteve correlacionado com a intensidade de uso de mecanização, sendo possivel auferir maiores margens brutas, tanto em sistemas totalmente mecanizados, quanto em sistemas com mecanização intermediária ou com tração animal. O custo com fertilizantes $e$ a produtividade foram os fatores mais importantes para $o$ desempenho econômico da cultura. Verificou-se também que o custo com sementes não esteve correlacionado com a margem bruta. Assim, para as condições do presente estudo, é economicamente mais interessante a utilização de materiais com alto potencial produtivo, mesmo que o custo de aquisição das sementes seja maior. Pela Análise de Cluster, as 26 situações culturais foram agrupadas em quatro classes de desempenho econômico. As menores margens brutas variaram entre $R \$ 180$ $h a^{-1}$ (sistema totalmente mecanizado) e $R \$ 288 \mathrm{ha}^{-1}$ (tração animal $e$ intermediário). As maiores margens brutas variaram entre $R \$ 553 \mathrm{ha}^{-1}$ (sistema totalmente mecanizado) e $R \$ 695 \mathrm{ha}^{-1}$ (tração animal e intermediário).

Palavras-chave: Helianthus annuus, análise multivariada, sistemas de produção, componentes principais, margem bruta.

\section{ABSTRACT}

This study aimed to evaluate the economic performance of sunflower in family farms of the Southeastern region of Paraná. Twenty-six situations were evaluated, according to the agroecological conditions, the different levels of mechanization, the amounts of fertilizer applied and varieties. Principal Component

\begin{abstract}
Analysis showed that the economic performance was not correlated to the level of mechanization. It was possible to obtain higher gross margins in intensive mechanization, intermediate or animal traction systems. Among the factors that comprise the gross margin, costs with fertilizers and crop yields were those of more importance for the economic performance. On the other side, as the seed costs was not correlated with the gross margin, for the conditions of this study, from the economic perspective it is more interesting the use of highyielding varieties, even if the cost is higher. ClusterAnalysis grouped the 26 situations into four classes of economic performance. The lowest gross margins ranged from $R \$ 180 \mathrm{ha}^{-1}$ (totally mechanized) and $R \$ 288 \mathrm{ha}^{-1}$ (intermediate and animal traction). The highest gross margins ranged from $R \$ 553 \mathrm{ha}^{-1}$ (totally mechanized) and $R \$ 695 \mathrm{ha}^{-1}$ (animal traction and intermediate).
\end{abstract}

Key words: Helianthus annuus, multivariate analysis, farming systems, principal component analysis, gross margin.

\section{INTRODUÇÃO}

O Programa Nacional de Produção e Uso de Biodiesel, aliado ao aumento da demanda internacional por óleos vegetais, abre novas oportunidades para a agricultura familiar. Dentre as espécies oleaginosas potenciais, a cultura do girassol destaca-se pelas suas características de ciclagem de nutrientes, baixo custo de produção e qualidades nutricionais. Por outro lado, vários estudos têm apontado para a baixa remuneração obtida pelos produtores, decorrente dos baixos preços recebidos e dos baixos rendimentos da cultura.

Instituto Agronômico do Paraná (IAPAR), Pólo Regional de Ponta Grossa, 84001-970, Ponta Grossa, PR, Brasil. E-mail:

fatima_ribeiro@iapar.br. Autor para correspondência.

IIDepartamento de Economia, Universidade Estadual de Ponta Grossa (UEPG), Ponta Grossa, PR, Brasil. 
Antes de se fomentar a disseminação de uma determinada tecnologia em uma região, necessita-se quantificar a sua contribuição potencial à melhoria da renda dos sistemas produtivos, bem como identificar e hierarquizar os fatores limitantes ao seu desempenho agronômico e econômico $\mathrm{Na}$ região sudeste paranaense, coexistem diferentes sistemas de produção familiar, desde aqueles baseados no uso intensivo da mão-de-obra familiar e da tração animal, até aqueles com uso mais intensivo de capital. Esperase, assim, que a cultura do girassol, implantada em diferentes situações culturais também apresente diferenças quanto aos indicadores de desempenho econômico. Nesse contexto, o presente trabalho tem como objetivos: a) quantificar o desempenho econômico da cultura do girassol em sistemas de produção familiar do sudeste paranaense e b) dentre as variáveis que compõem o desempenho econômico da cultura, identificar aqueles que mais contribuíram para a variabilidade dos valores observados.

\section{MATERIAL E MÉTODOS}

O estudo foi conduzido em 13 estabelecimentos familiares representativos dos principais sistemas de produção da região sudeste paranaense, nos quais foram implantadas 26 situações culturais (Tabela 1). Uma situação cultural é definida por JOUVE (1990) como um talhão homogêneo do ponto de vista biofísico e das técnicas aplicadas.

A tabela 1 apresenta a variabilidade das situações estudadas, no que se refere às cultivares utilizadas, à quantidade de sementes, à adubação (definida a partir dos teores de fósforo e potássio no solo e com base nas recomendações de CASTRO \& OLIVEIRA (2005) e às modalidades de mecanização. Maiores detalhes dos itinerários técnicos são descritos em RIBEIRO et al. (2011). Ressalta-se que, em todas as situações culturais, realizou-se apenas um controle mecânico de plantas daninhas na entrelinha.

Tabela 1 - Caracterização das situações culturais estudadas, safras 2008 e 2009

\begin{tabular}{|c|c|c|c|}
\hline Situação & Mecanização $^{1}$ & Cultivar & Doses de $\mathrm{N}-\mathrm{P}_{2} \mathrm{O} 5-\mathrm{K}_{2} \mathrm{O}\left(\mathrm{kg} \mathrm{ha}^{-1}\right)$ \\
\hline 1 & Tração animal & 'BRS $122 '$ & $40-40-40$ \\
\hline 2 & Tração animal & ${ }^{\circ}$ Helio 250' & $40-40-40$ \\
\hline 3 & Totalmente Mecanizado & 'BRS $122 '$ & $40-30-20$ \\
\hline 4 & Totalmente Mecanizado & ${ }^{\circ}$ Helio $250{ }^{\prime}$ & $40-30-20$ \\
\hline 5 & Totalmente Mecanizado & 'BRS 122' & $40-30-20$ \\
\hline 6 & Totalmente Mecanizado & ${ }^{\prime}$ Helio 250' & $40-30-20$ \\
\hline 7 & Intermediário & ${ }^{\circ} \mathrm{BRS} 122{ }^{\prime}$ & $40-60-20$ \\
\hline 8 & Intermediário & ${ }^{\circ}$ Helio $250 '$ & $40-60-20$ \\
\hline 9 & Intermediário & 'BRS 122 ' & $40-40-40$ \\
\hline 10 & Intermediário & ${ }^{\circ}$ Helio $250{ }^{\prime}$ & $40-40-40$ \\
\hline 11 & Tração animal & 'BRS 122 ' & $40-40-40$ \\
\hline 12 & Tração animal & ${ }^{\circ}$ Helio 250' & $40-40-40$ \\
\hline 13 & Intermediário & 'BRS 122 ' & $40-40-40$ \\
\hline 14 & Intermediário & ${ }^{\prime}$ Helio $250 '$ & $40-40-40$ \\
\hline 15 & Intermediário & ${ }^{\circ} \mathrm{BRS} 122$ ' & $40-30-20$ \\
\hline 16 & Intermediário & ${ }^{\circ}$ Helio $250{ }^{\prime}$ & $40-30-20$ \\
\hline 17 & Totalmente Mecanizado & 'CATISSOL' & $40-60-40$ \\
\hline 18 & Totalmente Mecanizado & 'CATISSOL' & $40-60-40$ \\
\hline 19 & Totalmente Mecanizado & 'CATISSOL' & $40-30-20$ \\
\hline 20 & Intermediário & 'CATISSOL' & $40-30-20$ \\
\hline 21 & Tração animal & 'CATISSOL' & $40-40-20$ \\
\hline 22 & Totalmente Mecanizado & 'CATISSOL' & $40-40-20$ \\
\hline 23 & Totalmente Mecanizado & 'CATISSOL' & $40-60-40$ \\
\hline 24 & Intermediário & 'CATISSOL' & $40-60-40$ \\
\hline 25 & Intermediário & 'CATISSOL' & $40-60-40$ \\
\hline 26 & Intermediário & 'CATISSOL' & $40-60-60$ \\
\hline
\end{tabular}

Tração Animal: preparo do solo, semeadura e controle de plantas daninhas com tração animal; adubação de cobertura e colheita manuais; Intermediário: preparo do solo mecanizado; semeadura mecanizada ou com tração animal; adubação de cobertura e colheita manuais; Totalmente Mecanizado: Preparo do solo, semeadura, adubação de cobertura, controle de plantas daninhas e colheita mecanizada.

Ciência Rural, v.43, n.5, mai, 2013. 
Em cada talhão, foram registrados os insumos (tipo e quantidade) e os equipamentos utilizados nas operações agrícolas (tipo, modelo e ano de fabricação), a demanda de mão-de-obra para as operações agrícolas e o rendimento da cultura. Esses dados foram utilizados para a determinação do custo de produção e da rentabilidade da cultura, segundo metodologia adotada por SOARES JR. \& SALDANHA (2000). Os indicadores de custo analisados foram o Custo Operacional Total (COT), o qual é constituído pelo Custo Operacional Efetivo (COE) e o Custo Fixo Total (CFT). O CFT correspondeu ao valor de depreciação dos equipamentos utilizados nas operações agrícolas. $\mathrm{O}$ COE compreendeu as despesas com insumos e as despesas com operações mecanizadas. Os valores de preços dos insumos, máquinas e implementos foram obtidos a partir das planilhas da SEAB/DERAL relativos ao mês de agosto de 2007 e 2008 e junto às revendas de insumos da região. $\mathrm{O}$ preço pago aos produtores pela saca de $60 \mathrm{~kg}$ de girassol foi informado pelo Núcleo regional da SEAB de Umuarama. Os valores obtidos para 2007 foram corrigidos para 2008 pela inflação, medida pelo IGP-DI da Fundação Getúlio Vargas.

Como variáveis de desempenho econômico, adotou-se a margem bruta (MB), a qual corresponde à diferença entre a renda bruta e o COT .

As relações entre as variáveis de desempenho econômico foram estudadas por meio de Análise de Componentes Principais (ACP), a qual consiste essencialmente em se reescrever as coordenadas das observações em outro sistema de eixos mais conveniente para a análise dos dados. Em outras palavras, as n-variáveis originais geram, através de suas combinações lineares, n-componentes principais, cuja principal característica, além da ortogonalidade, é que são obtidas em ordem decrescente de máxima variância, ou seja, a componente principal 1 detém mais informação estatística que a componente principal 2, que, por sua vez, detém mais informação estatística que a componente principal 3, e assim por diante. Destacase que a ACP pode ser usada para julgar a importância das próprias variáveis originais escolhidas, ou seja, as variáveis originais com maior peso (loadings) são as mais importantes do ponto de vista estatístico (CROCCO et al., 2003).

Para efetuar a ACP, construiu-se uma matriz constituída por seis variáveis e 26 observações, sendo cinco variáveis ativas (custo com fertilizantes, custo com sementes, custo com operações, rendimento de aquênios e demanda de mão-de-obra), e uma variável suplementar (margem bruta). O uso de variáveis suplementares em ACP é um recurso que permite que algumas variáveis participem da análise, sem participarem na construção dos eixos, e cuja variabilidade pode ser explicada pelas componentes com as quais estejam correlacionadas (TUFFÉRY, 2007). O critério para extração das componentes principais obedeceu ao valor mínimo de $75 \%$ da variância acumulada (MINGOTI, 2007). Posteriormente, utilizando-se a Análise de Agrupamento ou Análise de Cluster, as observações foram agrupadas utilizando-se a distância euclidiana como medida de distância entre os pontos e o método de Ward como algoritmo para o agrupamento. Essa análise tem como objetivo classificar as observações, de forma que os elementos pertencentes a um mesmo grupo sejam similares entre si com respeito às variáveis (características) que neles foram medidas, e os elementos em grupos diferentes sejam heterogêneos em relação a essas mesmas características (MINGOTI, 2007).

\section{RESULTADOS E DISCUSSÃO}

Verifica-se, pela tabela 2, que a demanda de mão-de-obra variou de $4 \mathrm{~h} \mathrm{ha}^{-1}$ na modalidade Totalmente Mecanizado, até $104 \mathrm{~h} \mathrm{ha}^{1}$ na modalidade Tração Animal. A colheita manual, presente nas modalidades Tração Animal e Intermediário, demandou, em média, 39h ha ${ }^{-1}$. O custo operacional total variou entre $\mathrm{R} \$ 451$, para a modalidade Tração Animal, e R\$ 943 para a modalidade Totalmente Mecanizado. Essa discrepância nos valores está atrelada diretamente às diferentes modalidades de mecanização, resultante da variabilidade no uso de capital entre os sistemas de produção estudados.

Em Santa Catarina, BORSUK (2008) avaliou os custos relativos ao cultivo do girassol na safrinha em 2007, implantada e conduzida por meio de operações motomecanizadas e com colheita manual (equivalente à modalidade Intermediário), por uma cooperativa de agricultores familiares. Para a produção de 1 ha de girassol, o custo operacional total variou de $\mathrm{R} \$ 502$ a $\mathrm{R} \$ 610$. Para um sistema totalmente mecanizado de implantação e condução da cultura do girassol no Mato Grosso do Sul, RICHETTI (2006) estimou um custo operacional efetivo de R $\$ 610$. Para a safra de 2008, RICHETTI (2008) estimou um custo operacional total de $\mathrm{R} \$ 737 \mathrm{ha}^{-1}$. Conforme relatos apresentados na XII Reunião Nacional de Pesquisa em Girassol (ALMEIDA, 2009), na safrinha de 2008, o custo variável para a produção de 1 ha de girassol no Estado de Goiás situou-se entre R $\$ 600$ e R\$926 


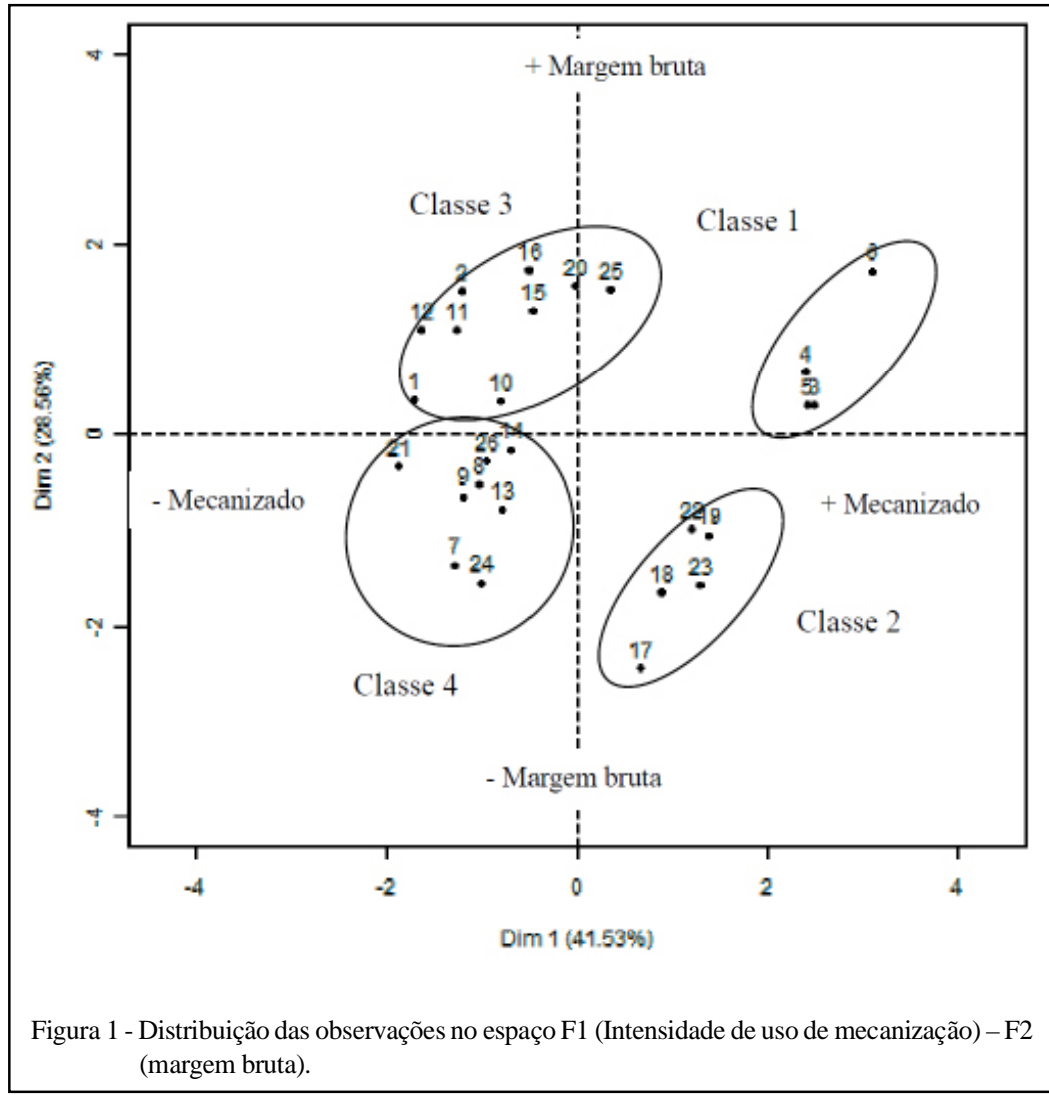

elevado $\left(1602 \mathrm{~kg} \mathrm{ha}^{-1}\right)$. A consequência direta foi então a obtenção do maior valor de margem bruta $\left(\mathrm{R} \$ 636 \mathrm{ha}^{-1}\right)$ dentre todos os grupos.

A Classe 4 é constituída pelas situações com baixas margens brutas e média intensidade de uso de mecanização, na qual predominam baixos rendimentos $\left(1211 \mathrm{~kg} \mathrm{ha}^{-1}\right)$.

Comparando-se as Classes 1 e 2, verificase que, no sistema Totalmente Mecanizado, as altas margens brutas foram condicionadas aos altos rendimentos e ao menor custo com fertilizantes. Porém, verifica-se que, na Classe 3 , também foi possível auferir altas margens brutas em situações de média produtividade, com a condição de baixo custo com fertilizantes e operações agrícolas.

Esses resultados mostram a possibilidade de se auferir margens brutas elevadas, independentemente do nível de mecanização utilizado, desde que duas condições sejam atendidas: a primeira, refere-se à utilização de práticas de manejo da cultura que permitam a obtenção de rendimentos acima de $1600 \mathrm{~kg} \mathrm{ha}^{-1} \mathrm{e}$ a segunda, que se invista em práticas de manejo da fertilidade do solo, de forma que o custo com a adubação não ultrapasse $\mathrm{R} \$ 307 \mathrm{ha}^{-1}$.

Tabela 4 - Descrição das classes de desempenho econômico segundo a análise de cluster, safras de 2007 e 2008.

\begin{tabular}{|c|c|c|c|c|}
\hline \multirow[t]{2}{*}{ Variáveis } & \multirow[b]{2}{*}{1} & \multirow[b]{2}{*}{2} & \multirow[b]{2}{*}{3} & \multirow[b]{2}{*}{4} \\
\hline & & & & \\
\hline Custo com fertilizantes $\left(\mathrm{R} \$ \mathrm{ha}^{-1}\right)$ & 307,09 & 394,23 & 307,02 & 384,50 \\
\hline Custo com operações ( $\mathrm{R} \$ \mathrm{ha}^{-1)}$ & 370,05 & 297,13 & 65,66 & 92,21 \\
\hline Rendimento de aquênios $\left(\mathrm{kg} \mathrm{ha}^{-1}\right)$ & 2044,30 & 1323,95 & 1602,00 & 1211,84 \\
\hline Demanda de mão-de-obra $\left(\mathrm{h} \mathrm{ha}{ }^{-1}\right)$ & 4,75 & 4,16 & 81,43 & 62,36 \\
\hline Margem bruta $\left(\mathrm{RS} \mathrm{ha}^{-1}\right)$ & 552,88 & 180,28 & 635,96 & 288,49 \\
\hline
\end{tabular}




\section{CONCLUSÃO}

Os resultados do presente estudo, conduzido em 26 situações de cultivo do girassol, caracterizadas por uma grande variabilidade, mostraram que o desempenho econômico da cultura não esteve correlacionado com a intensidade de uso de mecanização, sendo possível auferir maiores margens brutas tanto em sistemas totalmente mecanizados quanto em sistemas com mecanização intermediária ou com tração animal. Dentre os fatores que compõem a margem bruta, o custo com fertilizantes e o rendimento da cultura foram os que mais contribuíram para o desempenho econômico. Por outro lado, como o custo com sementes não esteve correlacionado à margem bruta, para as condições em que foi realizado este trabalho, economicamente, é mais interessante a utilização de materiais com alto potencial produtivo, mesmo que o custo de aquisição das sementes seja maior.

\section{AGRADECIMENTOS}

À Coordenação de Aperfeiçoamento de Pessoal de Nível Superior (CAPES), pela concessão da bolsa de estágio de doutorado no exterior, ao Ministério do Desenvolvimento Agrário (MDA) e à Petrobrás/SIX, pelo apoio financeiro para a realização do projeto.

\section{REFERÊNCIAS}

ALMEIDA, I.R. Relatos do comportamento da cultura do girassol por região. In: REUNIÃO NACIONAL DE PESQUISA DE GIRASSOL, 18.; SIMPÓSIO NACIONAL SOBRE A CULTURA DO GIRASSOL, 6., 2009, Pelotas, RS. Atas e Resumos... Pelotas: EMBRAPA Clima Temperado, 2009. 1 CD-ROM.

BORSUK, L.J. Cultivo de girassol sob a perspectiva da agroenergia: uma análise dos produtores familiares assentados de Abelardo Luz, SC. 2008. 92f. Dissertação (Mestrado em
Agrossistemas) - Universidade Federal de Santa Catarina, Florianópolis, SC.

CASTRO, C.; OLIVEIRA, F.A. Nutrição e adubação do girassol. In: LEITE, R.M.V.B.C. et al. (Ed.). Girassol no Brasil. Londrina: EMBRAPA Soja, 2005. p.317-373.

JOUVE, P. Analyse agronomique de situations culturales. In: Agronomie et ressources naturelles en régions tropicales: JOURNEES SUR L'AGRONOMIE ET LES RESSOURCES NATURELLES EN REGIONS TROPICALES, 15., 1990, Montpellier. Actes des journeés... Montpellier: CIRAD-IRAT, 1990. p.127-135.

MINGOTI, S.A. Análise de dados através de métodos de estatística multivariada: uma abordagem aplicada. Belo Horizonte: UFMG, 2007. 300p.

RIBEIRO, M.F.S. et al. Desempenho agronômico da cultura do girassol em diferentes condições edafoclimáticas do Sudeste paranaense. Bragantia, v.70, n.3, p.550-560. 2011. Disponível em: <http://www.scielo.br/scielo.php?script=sci_arttext\&pid=S0006$87052011000300010 \& \ln \mathrm{g}=\mathrm{en} \& \mathrm{nrm}=\mathrm{iso}>$. Acesso em 28 maio, 2012. doi: http://dx.doi.org/10.1590/S0006-87052011000300010.

RICHETTI, A. Estimativa do custo de produção de girassol em Mato Grosso do Sul, safra 2007. Dourados: EMBRAPA Agropecuária Oeste, 2006. p.1-2. (Comunicado Técnico, n.130).

RICHETTI, A. Estimativa de custo de produção de girassol em Mato Grosso do Sul, safra 2008. Dourados: EMBRAPA Agropecuária Oeste, 2008. p.1-2. (Comunicado Técnico, n.142).

SOARES JUNIOR, D.; SALDANHA, A.N.K. Indicadores econômicos propostos para a análise de sistemas de produção e propriedades agropecuárias trabalhadas nas Redes de Referências para a Agricultura Familiar. In: SEMINÁRIO ESTADUAL DE ADMINISTRAÇÃO RURAL, 5.; SEMINÁRIO SUL BRASILEIRO DE ADMINISTRAÇÃO RURAL, 1, 2000, Itajaí, SC. Anais... Itajaí: Associação Brasileira de Administração Rural, 2000. Disponível em: <http://www.iapar.br/arquivos/File/zip_pdf/ redereferencia/a_indic_econo.pdf $>$. Acesso em: 20 abr. 2010.

TUFFÉRY, S. Data mining et statistique décisionelle: l'inteligence des données. Paris: Technip, 2007. 481p. 